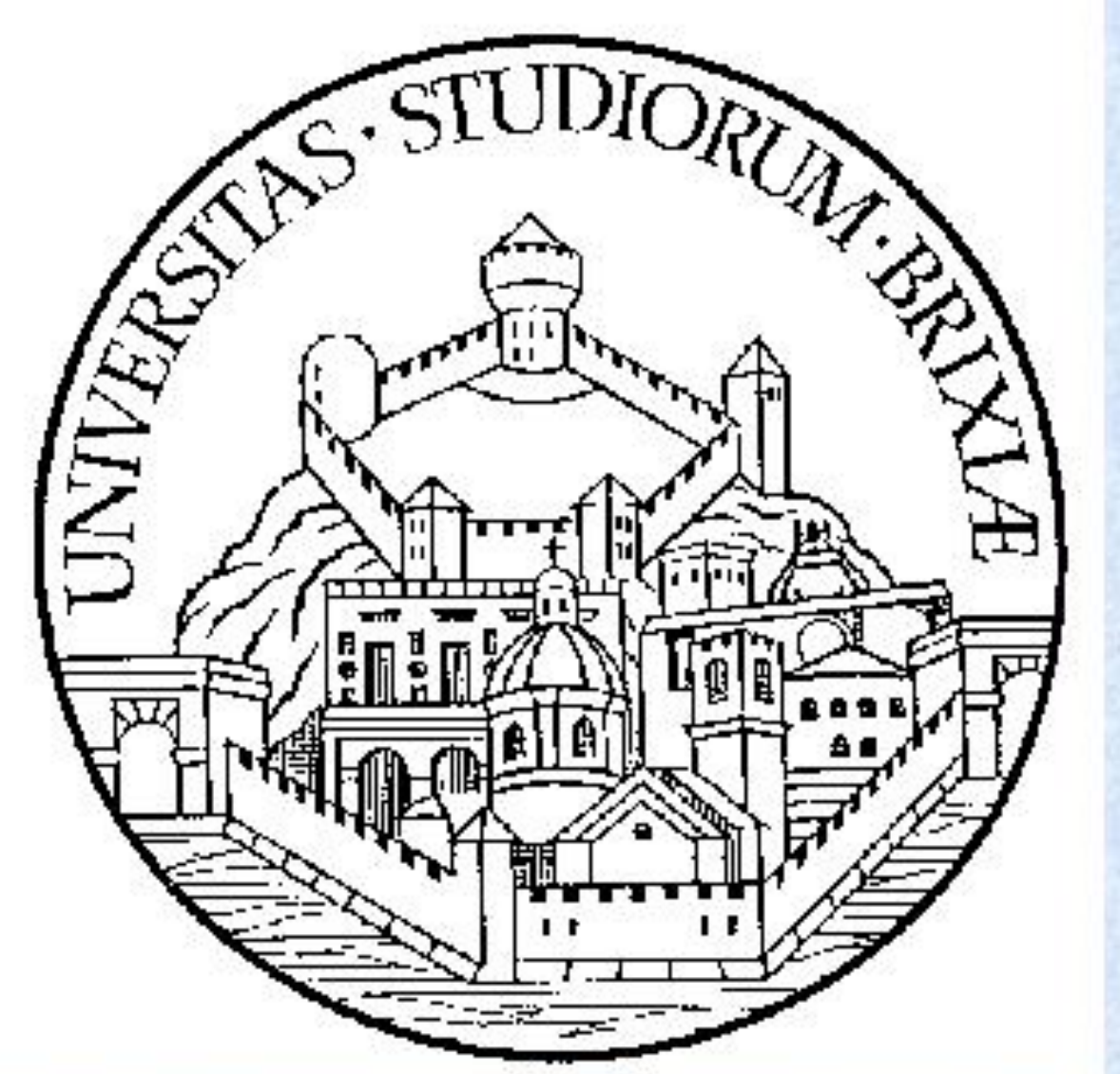

\title{
The effects of pituitary replacement therapies on body composition in adult patients with growth hormone deficiency.
}

\author{
S. Frara ${ }^{1}$, F. Maffezzoni ${ }^{1}$, A.M. Formenti ${ }^{1}$, M. Mezzone ${ }^{1}$, C. Ronchini Ferreira ${ }^{1}$, F. Doglietto ${ }^{2}$, R. Maroldi ${ }^{3}$, G. Mazziotti ${ }^{1,4}$, A. Giustina $^{1}$ \\ ${ }^{1}$ Endocrinology and Metabolic Disease Unit, Department of Molecular and Translational Medicine University of Brescia; ${ }^{2}$ Neurosurgery Unit, ASST Spedali Civili, Brescia; \\ ${ }^{3}$ II Radiology Unit, ASST Spedali Civili, Brescia; ${ }^{4}$ Internal Medicine Department, ASST Carlo Poma, Mantova.
}

\section{Background}

GH Deficiency (GHD) has been clearly associated with adverse metabolic profiles (e.g. glycemic impairment, low HDL-cholesterol, high triglycerides and LDLcholesterol) as well as osteoporosis with high risk of pathological fractures, reduced life expectancy and poor quality of life.

In this clinical setting, characterized by altered body composition and adverse cardiovascular risk, the effects of other pituitary hormone deficiencies or their replacement therapies on the metabolic profile are still a matter of debate.

In fact, it is well known that excess of hydrocortisone acts increasing fat mass and reducing lean tissues. On the other hand, high dosages of L-T4 may reduce total body fat mass inducing its catabolism.

\section{Aim}

Purpose of our study was to evaluate the effects of replacement therapies of central hypoadrenalism and hypothyroidism on body composition in a cohort of 33 patients (F 7, M 26) with treated and untreated GHD.

Patients \& Methods

\begin{tabular}{l|c|} 
& Patients \\
\hline Sex (M vs F) & 26 vs 7 \\
\hline Median age (years) & $51(18-77)$ \\
\hline Patients treated for GHD & 10 \\
\hline Patients treated for hypothyroidism & 25 \\
\hline L-T4 median daily dose pro Kg & $1.27(0.4-2.6) \mathrm{mcg} / \mathrm{Kg} / \mathrm{day}$ \\
\hline Patients treated for glucocorticoid deficiency & 22 \\
\hline Hydrocortisone median daily dose & $25(10-40) \mathrm{mg} / \mathrm{die}$
\end{tabular}

Patients were evaluated for their anthropometric measures and body composition by Hologic Discovery DXA machine.

\section{Results}

$\checkmark$ Subjects with untreated GHD showed higher total body fat percentage (30.9\% range: $22-52$ vs. $23.0 \%$, range: $11-42 ; p=0.03$ ) and waist circumference (105 cm vs. $88 \mathrm{~cm}$; $p=0.009$ ) (Fig. 1) as compared to patients with treated GHD.

$\checkmark$ However, no significant differences in body composition were observed between patients with treated hypoadrenalism and those with preserved adrenal function (total body fat percentage: $30.6 \%$, range $11-52$ vs. 26.8 , range 13-45; $p=0.48$; waist circumference: $98.5 \mathrm{~cm}$, range $75-130$ vs. $103.0 \mathrm{~cm}$, range: $75-140 ; p=0.98$ ) (Fig. 2). $\checkmark$ No significant differences were observed between patients treated with L-T4 and those with normal thyroid function (total body fat percentage: $29.6 \%$, range $11-52$ vs. 30.4 , range $13-45 ; p=0.82$; waist circumference: $99.0 \mathrm{~cm}$, range $75-130$ vs. $98.0 \mathrm{~cm}$, range: $75-140 ; p=0.91$ ) (Fig. 3).

$\checkmark$ Moreover, daily dose of hydrocortisone did not correlate with total body fat percentage (rho: $0.01 ; p=0.9$ ) and waist circumference (rho: 0.29; $\mathrm{p}=0.27)$.

$\checkmark$ Likewise, no significant associations were found between L-T4 daily dose and total body fat percentage (rho: $0.17 ; p=0.4$ ) and waist circumference (rho: $0.25 ; \quad p=0.30$ ) in patients with central hypothyroidism.
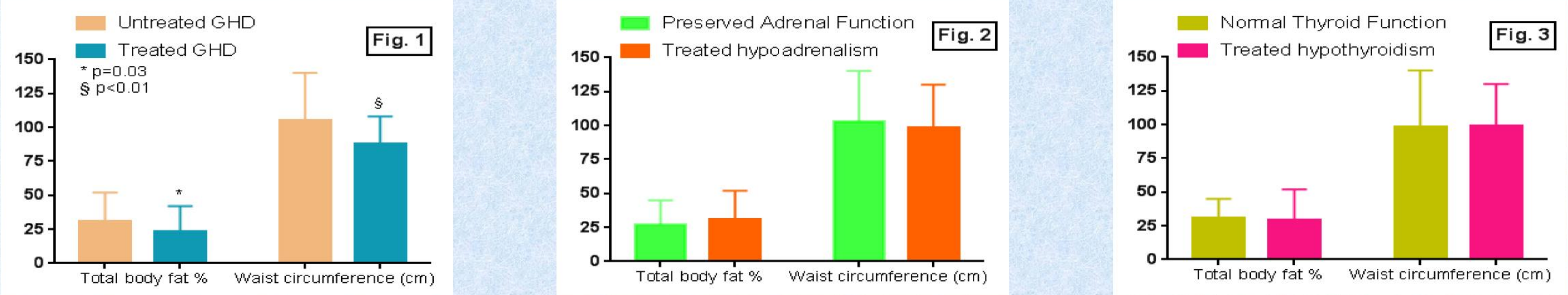

\section{Conclusions}

$\checkmark$ This study suggests that GHD is the main determinant of body composition in adult hypopituitary patients.

$\checkmark$ In fact, replacement therapies with hydrocortisone and L-T4 (when prescribed in appropriate doses) do not seem to exert relevant effects on this clinical outcome in this setting. 